Open Access

\title{
PHEDRE trial protocol - observational study of the prevalence of problematic use of Equimolar Mixture of Oxygen and Nitrous Oxide (EMONO) and analgesics in the French sickle-cell disease population
}

\author{
Marie Gérardin ${ }^{1,2 *}$, Marie-Laure Couec ${ }^{3}$, Marie Grall-Bronnec ${ }^{2,4}$, Fanny Feuillet ${ }^{2,5}$, Laura Wainstein ${ }^{1}$,
} Morgane Rousselet ${ }^{1,2,4}$, Marie-Lyne Pinot ${ }^{1}$, Fanny Perrouin ${ }^{1}$, Olivier Bonnot $^{6}$, Marie-Hélène Drouineau ${ }^{7}$, Pascale Jolliet ${ }^{1,2}$ and Caroline Victorri-Vigneau ${ }^{1,2}$

\begin{abstract}
Background: The use of analgesics can lead to cases of drug abuse and dependence. It can also cause pseudoaddiction in patients suffering from pain. What is the actual situation in patients suffering from severe sickle-cell disease, exposed to acute pain during vaso-occlusive crises? Evaluation of the use of analgesics, on the basis of Diagnostic and Statistical Manual of Mental Disorders criteria for substance abuse and dependence, makes it possible to differentiate the symptoms occurring only in a context of pain, in the aim of managing the pain, and thus describing pseudo-addiction, from symptoms also occurring when there is no pain, and more in favour of true addiction. Currently there is no data available in France on this problem, and no studies have been carried out in children or adolescents with sickle-cell disease. The purpose of the study is to evaluate the prevalence of problematic use of equimolar mixture of oxygen and nitrous oxide and other analgesic drugs in a population of subjects with severe sickle-cell disease in France.
\end{abstract}

Methods/design: PHEDRE (Pharmacodépendance Et DREpanocytose-drug dependence and sickle-cell disease) is an observational, descriptive and transversal study. Patients under the age of 26 with sickle-cell disease are included in the study by the doctors looking after them in sickle-cell disease centres. The patients are then contacted by a trained researcher for a telephone interview, including an evaluation of the Diagnostic and Statistical Manual of Mental Disorders criteria for abuse and dependence to equimolar mixture of oxygen and nitrous oxide and for each of the analgesic drugs taken by the patient. The data are also completed using the subject's medical record.

(Continued on next page)

\footnotetext{
* Correspondence: marie.gerardin@chu-nantes.fr

${ }^{1}$ Clinical Pharmacology Department, Nantes University Hospital, Institut de Biologie, 9 quai Moncousu, 44093 Nantes Cedex 1, France

${ }^{2}$ EA 4275 SPHERE « bioStatistics, Pharmacoepidemiology and Human sciEnces REsearch, Faculties of Medicine and Pharmaceutical Sciences, Nantes University, Nantes, France

Full list of author information is available at the end of the article
}

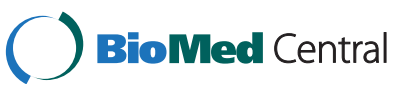

(c) 2015 Gérardin et al. Open Access This article is distributed under the terms of the Creative Commons Attribution 4.0 International License (http://creativecommons.org/licenses/by/4.0/), which permits unrestricted use, distribution, and reproduction in any medium, provided you give appropriate credit to the original author(s) and the source, provide a link to the Creative Commons license, and indicate if changes were made. The Creative Commons Public Domain Dedication waiver (http://creativecommons.org/publicdomain/zero/1.0/) applies to the data made available in this article, unless otherwise stated. 
(Continued from previous page)

Discussion: This study will make it possible to provide an initial quantitative and qualitative evaluation of problematic use of equimolar mixture of oxygen and nitrous oxide and analgesic drugs in the sickle-cell disease population. The results will be used firstly to provide additional data essential for monitoring the risk of overdose, abuse, dependence and misuse of these products, and to begin awareness-raising and to provide information for health care professionals, in order to significantly improve the management of sickle-cell disease-related pain.

Trial registration: Clinical Trials.gov ID: NCT02580565 registered 16 October 2015

Unique Protocol ID: RC14_0344

Keywords: Sickle-cell disease, Equimolar Mixture of Oxygen and Nitrous Oxide (EMONO), Analgesic drugs, Problematic use, Pseudo-addiction, Substance use disorders

\section{Background}

Equimolar Mixture of Oxygen and Nitrous Oxide (EMONO) is a gas mixture that contains equal parts of each. It has Marketing Authorization (MA) in France since 2001. It is especially indicated in short-term analgesia for painful procedures or in the event of mild to moderate pain in adults and children over the age of one month.

Dependence on and tolerance to the effects of nitrous oxide have been demonstrated in animals and in humans [1-4]. Numerous cases of nitrous oxide abuse having led to adverse effects have been reported in literature [5-18]. Variability in the effects experienced with nitrous oxide is described in numerous studies [19], ranging from dysphoria to euphoria. Concerning EMONO, there is little literature on the potential of abuse, as few countries have the mixture as a drug. In the United States, the gas is delivered by pharmaceutical companies to professionals, who then perform titration themselves. Cases of severe abuse and dependence to EMONO have however been declared to the French addiction monitoring system via the network of Centres for the Evaluation of and Information on drug Dependence (Centres d'Evaluation et d'Information sur la Pharmacodépendance - CEIP-A). Analysis of these cases showed that it concerned patients in a serious medical situation, requiring repeated use of EMONO in hospital, and that a certain number of these subjects suffered from severe Sickle-Cell Disease (SCD). It therefore appeared that the SCD population could represent a high risk population for EMONO abuse or dependence. Thus, a specific data collection in these subjects was needed.

Sickle-cell anaemia and severe SCD are haemoglobinopathies from the group of corpuscular hereditary haemolytic anaemias. In France, patients with SCD are managed via a patient-oriented care network, especially Reference Centres or Special Centres for Children and Adults (RSCCA). These centres are scientific and clinical excellence structures for rare diseases such as SCD. The RSCCA medical practitioners see each patient, regardless of their age, at an inpatient/or outpatient consultation, at least once per year as part of routine follow-up. The acute pain caused by Vaso-Occlusive Crises (VOC) are the most common clinical symptom and the first cause that lead to the emergency department for children, adolescents and adults with SCD. Patients are also prone to chronic or recurrent acute pain, that require analgesics taken at the patient's home. This pain is subject to significant interindividual variability but also intra-individual variability, as much in terms of severity, site and duration of the pain episode [20,21]. The unforeseeable nature of the pain and extent of the active pain relief strategies (called coping strategies), developed by patients with SCD, are different from other painful disorders in which similar analgesic treatment is used, and imply the difficulties patients and health care professionals experience in managing it $[21,22]$. According to the intensity of the pain and type of treatment and according to the World Health Organisation's (WHO) classification, level I analgesics, (acetaminophen and Non-Steroidal Anti-Inflammatory drugs (NSAIDs)), level II (codeine, tramadol, nalbuphine) and level III (morphine) can be used. EMONO is ideal for the rapid management of pain and/or in combination with morphine in hospital in children and adolescents [21,23]. One of the barriers to management of pain in patients with SCD is the reticence of health care professionals to use morphine-based analgesics, for fear of the patient developing drug dependence [21]. Indeed, the request for analgesics can be perceived, not as a response to the pain episode but as a drug addiction [24, 25], whereas most often it is a simple clinical impression [26]. Nevertheless, undertreated pain exposes patients to the risk of "pseudoaddiction", which occurs when patients attempt to obtain a stronger analgesic dose, thus possibly coming across resistance from the nursing team. These behaviours, which look like the compulsive search for analgesic drugs, are interpreted as being addictive-like-behaviour and widely keep the beliefs of the healthcare team as to the dependence of their patients. However in pseudo-addiction, analgesic-seeking behaviour stops when the pain is treated effectively and does not persist after pain relief has been 
achieved [27]. Evaluation of the use of analgesic drugs, on the basis of Diagnostic and Statistical Manual of Mental Disorders (DSM) criteria for substance abuse and dependence [28], makes it possible to differentiate the symptoms occurring only in a context of pain, in the aim of managing the pain, and thus describing pseudo-addiction (common), from symptoms also occurring when there is no/mild pain, and more in favour of true addiction (rarer). The major risk is that these patients who, in order to deal with their pain, demonstrate behaviour compatible with substance use disorder criteria, receive fewer analgesics and in whom therefore treatment is not optimal [29-31]. Also, if genuine drug addiction to analgesics was identified, it would require treatment adjustment. A specific evaluation is therefore necessary in order to improve patients' management. The role of EMONO and opiate analgesics in the treatment strategy, the potential for abuse and dependence to which is well known, today require special monitoring of the risk of overconsumption, abuse and dependence in this population.

Currently there are no data available in France on this concern, and no studies have been carried out in children or adolescents with SCD. This lack of data is all the more significant when we know that childhood experiences and those in adolescence can determine emotions and behaviours and strategies for adaptation to events in adulthood. The main objective of this study is to evaluate the prevalence of problematic use of EMONO in a population of subjects with severe SCD treated in RSCCA in France. The secondary objectives are:

- To evaluate the prevalence of problematic use of other WHO level II and III analgesic drugs used in the treatment of pain in patients with SCD treated in RSCCA in France.

- To describe and to characterize problematic use of EMONO and other analgesic drugs.

- To study the relationships between the existence of problematic use of analgesic drugs (EMONO and/or other analgesics) and the other study evaluation parameters (sociodemographic, clinical and therapeutic data and consumption of other psychoactive substances).

\section{Methods/Design Study design}

It is an observational, descriptive, transversal study aiming to determine the prevalence of problematic use of EMONO and other analgesic drugs in subjects with SCD treated in RSCCA. Subjects with SCD are recruited by medical practitioners of RSCCA. Thereafter, data are collected by a scheduled telephone interview and with subject's medical record.
This project received the financial backing from the French National Agency for Medicines and Health Products Safety (Agence Nationale de Sécurité du Médicament et des produits de santé - ANSM) as part of the 2014 'Young Scientist' request for proposals.

\section{Setting of the study}

The CEIP-A in Nantes, is the coordinating investigation centre in charge of the general management, monitoring and coordination of the project. A consortium was set up with the partner CEIP-A in charge of coordinating the project at local level in their research area (CEIP-A in Clermont-Ferrand, Lille, Marseille, Paris and Toulouse). The RSCCA participating in the study are spread over metropolitan France territory. The RSCCA medical practitioners are in contact with the CEIP-A in Nantes and with the CEIP-A in their area. The period of inclusion for each participating medical practitioner is 12 months and the period of data collection is 15 months. There is no follow up after the patient's assessment.

For this national multicentric study, a multi-disciplinary steering committee, comprising pharmacologists from the participating CEIP-A, medical practitioners working in RSCCA, a methodologist biostatistician, a clinical research staff and a psychiatrist specialising in addiction was set up in order to draft the study protocol. Its missions are to ensure the study is valid in terms of scientific content and methodology. The steering committee should handle any practical problems occurring during the study and where appropriate, decide on amendments to the protocol. It shall meet on a regular basis and whenever necessary, to approve aspects concerning practical organisation and to answer any questions raised during the study.

\section{Participants Eligibility criteria}

The patients are recruited for the study by their doctor when they come to the RSCCA for their routine treatment or follow-up, according to the following inclusion criteria:

- Subject with confirmed SCD diagnosis, regardless whether it is the homozygous or heterozygous form

- Subject treated for SCD at a RSCCA participating in the study, regardless of the duration of the illness

- Subject under the age of 26

- Written consent from adult subjects and written consent from one of the parents or legal guardians of minors (under 18)

The study exclusion criteria are the following:

- State-protected adult (under guardianship) 
- Subject not having the general aptitude to participate in the study assessment (i.e. not able to respond to the telephone interview): too young, insufficient motor development, major difficulties understanding the French language and/or speech and/or hearing disorders.

\section{Patient recruitment}

Each medical practitioner in the RSCCA participating in the study recruits SCD patients over a 12-month period. If the patient meets all inclusion criteria, the medical practitioner asks him, and his parents or legal guardian if the subject is a minor, if he wishes to participate in the study, and provides clear and appropriate verbal information on the study (objectives, scientific interest, and practical procedures).

- After a cooling-off period, he collects the patient's written consent (and from at least one of the parents or the legal guardian for minors).

To facilitate schedule of the telephone interview and the collection of study data during the telephone interview, the patient has to complete

two additional forms:

- The "inclusion form" is completed directly with the medical practitioner during the outpatient/ inpatient consultation. This form contains identification of the RSCCA, name of the medical practitioner, name of the patient, phone number, birthdate, moment when patient can be called (days, hours). The inclusion form and written consent will be sent at the same time by secure messaging to the Nantes CEIP-A by the medical practitioner.

- The "analgesic list form" is completed by the patient alone or/and with his family (for minors) after the medical consultation but before the telephone interview. The patient should complete the form with all analgesic drugs he has taken (at home and in hospital) over the last 12 months (names and frequencies).

- Then, the medical practitioner has to complete a short clinical assessment.

The inclusion form and written consent are triplicate documents. One copy is given to the patient, one is sent by the medical practitioner to the Nantes CEIP-A, and one is kept in the RSCCA. The clinical assessment completed by the medical practitioner is also kept in the RSCCA. Once included in the study, patients will undergo a single assessment by phone, without subsequent follow-up.

\section{Consent for participation}

At inclusion, the patients receive an information leaflet for their age range (as do the parents of minors). Then, they sign a consent form. For minors, parents also sign a consent form authorizing their children's participation.

\section{Ethics statement}

This non-interventional study received authorisation from the French data protection body (Commission Nationale de l'Informatique et des Libertés - CNIL) and a favourable opinion from the French advisory board for data processing in health care (Comité Consultatif sur le Traitement de l'Informatique en matière de Recherche dans le domaine de la Santé - CCTIRS) and from the local ethics committee (Groupe Nantais d'Ethique dans le Domaine de la Santé - GNEDS).

\section{Variables and data measurement}

Data for the analysis are collected in two steps: through the clinical assessment completed by the medical practitioner and through a single telephone interview.

\section{Clinical assessment}

After he includes a patient, the medical practitioner completes a clinical assessment which contains all information about patient's family and personal history, history of the SCD and treatment already received for SCD. Every six months, the trained researcher will schedule a visit to the RSCCA to retrieve the clinical assessments of all patients included in this RSCCA and enter the data in the electronic case report form (e-CRF).

\section{Telephone interview}

When Nantes CEIP-A receipts the inclusion form and written consent, the trained researcher schedules a telephone interview with the patient. Telephone interview is carried out with a trained researcher from Nantes CEIP-A. The interview is suited to the child's, adolescent's or young adult's age, maturity and comprehension skills. During the telephone interview, data are stored in the electronic case report form (e-CRF). The following parameters are evaluated during the telephone semi-structured interview for EMONO, and for other analgesic drugs, during pain episodes and outside pain episodes:

\section{Prevalence of problematic use:}

Problematic use is abuse of or dependence on a substance according to the fourth edition of DSM (DSMIV), and substance use disorder according to the fifth edition (DSM 5). At the time the protocol was written, we used DSM-IV criteria because the DSM 5 was not yet translated in French. The DSM-IV identifies seven substance dependence diagnostic criteria and four substance abuse diagnostic criteria. Abuse is defined by at least one of the four DSM-IV criteria of substance abuse 
and drug dependence by at least three of the seven DSM-IV criteria of substance dependence.

\section{Description and characterisation of problematic use:}

Consumption will be evaluated on the basis of quantitative and qualitative responses on the effects sought, quantitative and qualitative responses to each substance abuse and dependence criterion, by separating the painrelated criteria and non-pain-related criteria and quantitative and qualitative responses on craving.

\section{Study of the relationships between problematic use and the other study evaluation parameters}

The outcome measures are sociodemographic, clinical and therapeutic data and data on consumption of other psychoactive substances, and their relationship with problematic use of analgesic drugs. This study will be used to demonstrate any factors relating to problematic use of analgesic drugs, or even a specific analgesic drug.

\section{Bias}

The main potential bias in this study is the heterogeneity bias during data collection. It has been prevented by the choice of a single evaluator.

\section{Study size}

This study has never been driven among children and teenagers with $\mathrm{SCD}$, which is a rare disease.

For this reason, and considering the low number of patients with SCD in France, we planned to include all those who meet the inclusion criteria in RSCCA that agreed to participate in the study. Then, we did not calculate a sample size. Before the beginning of the study, the partner CEIP-A were asked to assess the number of patients fulfilling the inclusion criteria, at their local level, in the RSCCA that agreed to participate. The total number of patients who could potentially be included in France was thus estimated at around 2000 people.

As the RSCCA medical practitioners see each patient at least once per year as part of routine follow-up, the period of inclusion was set at 12 months, to ensure that the study would be proposed to all eligible patients.

\section{Statistical methods}

All evaluation parameters will be subjected to a descriptive analysis. The quantitative variable evaluation parameters will be described using position parameters (mean or median) and dispersion (standard deviation, interquartile range, range). Normality of their distribution will be assessed (normality test and evaluation using the relevant graphic tools). The qualitative variable evaluation parameters will be shown in the form of number and frequency tables for each modality. The primary outcome measure will be evaluated by the frequency of subjects with EMONO problematic use compared to the overall study population. The secondary outcome measures will be assessed by the frequency of subjects demonstrating problematic use of one or several other analgesic drugs, compared to the overall study population, by estimating the occurrences of positive responses for the various effects sought when taking analgesic drugs, for each DSM-IV substance abuse and dependence criterion, for each pain-related criterion, and non pain-related criterion (if the DSM-IV substance abuse and dependence criteria are met), and for craving. The frequencies will be estimated for EMONO and for each of the other analgesic drugs, the use of which is identified as problematic. The relationships between problematic use of analgesic drugs (EMONO and/or others) and the other study evaluation parameters will be assessed by univariate analysis and the appropriate statistics tests, depending on the type of variable and the size of the study population (parametric or non-parametric tests). The existence or absence of problematic use can then be studied as a dependent variable in a multivariate analysis (i.e. logistic regression) in order to test the relationship with independent variables selected from the previous analysis. No intermediate analysis or subgroup analysis is to be carried out in this study.

\section{Discussion}

Recruiting patients with SCD, which is a rare disease, is a challenge for the partners working on the project. The selection criteria further restrict the number of eligible patients. Due to feasibility concerns, the entire active file of children, adolescents and young adults with SCD meeting the inclusion criteria at each participating RSCCA will be included over a 12-month period.

The purpose of the study is to evaluate problematic use of analgesic drugs, received and/or taken at the hospital and at home. Such consumption is not always noticed or identified by the subject's friends, family or doctor. It is therefore essential to question subjects directly about this concern, without the opinion of the prescribing physician or family, and without interfering in the doctor-patient relationship. This evaluation takes place during a semi-structured interview with the subject. The inclusion criteria will have first made it possible to select only those subjects able to respond to the assessment. Given the variability in the study population, the interview will nevertheless be adapted to the child's, adolescent's or young adult's age, maturity and comprehension skills. If necessary, for minors, and in order to only collect certain sociodemographic or clinical data or data relating to medicinal product consumption 
(not on the problematic aspect of consumption), the subject's parent or legal guardian may also be questioned in addition to the subject.

For this multicentric study, centralised data collection by a single evaluator is necessary in order to prevent heterogeneity bias. Data collection during a scheduled telephone interview is used, on the one hand, to protect the confidentiality of the subject's data, and on the other hand, to ensure the quality of the data collected as the subjects selected will be available. This type of consumption data (cigarettes, medicinal products, drugs etc.) collection by telephone has already been seen in a number of national surveys, such as the Baromètre Santé by the French national institute for health prevention and education (Institut National de Prévention et d'Education pour la Santé - INPES), in which subjects were compliant and the results were of good quality.

The Nantes CEIP-A is competent in the assessment of drug dependence and has recognised expertise in this field. It has conducted several studies evaluating consumption of psychoactive substances. This positive experience confirms the feasibility of these assessment methods for the Nantes CEIP-A and will therefore ensure the collection of quality data in the PHEDRE project.

The use of DSM-IV criteria could be seen as a limitation since the DSM 5 [32] is now translated and therefore usable in French. But we initially choose to assess both DSM-IV dependence and abuse criteria in addition with craving and finally, this set of criteria is consistent with the DSM 5 substance use disorders diagnostic criteria.

PHEDRE is the first study which proposes to evaluate problematic use of EMONO and analgesics in the French SDC population. We believe that differentiating pseudo-addiction from real substance use disorder will enable clinicians to adapt patient management: increase analgesia in pseudo-addiction, provide an addictological follow-up for the others.

\footnotetext{
Abbreviations

ANSM: Agence Nationale de Sécurité du Médicament et des produits de santé (French health products agency); CCTIRS: Comité Consultatif sur le Traitement de I'Informatique en Matière de Recherche dans le Domaine de la Santé (French advisory board for data processing in healthcare); CEIPA: Centre d'Evaluation et d'Information sur la Pharmacodépendance (Centre for the evaluation of and information on drug dependence and addiction monitoring); CNIL: Commission Nationale de l'Informatique et des Libertés (French data protection body); DSM IV: Diagnostic and Statistical Manual of Mental Disorders (4 ${ }^{\text {th }}$ revision); DSM 5: Diagnostic and Statistical Manual of Mental Disorders (5 ${ }^{\text {th }}$ revision); e-CRF: Electronic Case Report Form; EMONO: Equimolar Mixture of Oxygene and Nitrous Oxide; GNEDS: Groupe Nantais d'Ethique dans le Domaine de la Santé (Nantes ethics committee); INPES: Institut National de Prévention et d'Education pour la Santé (French national institute for health prevention and education); MA: Marketing Authorization; NSAID: Non-Steroidal Anti-Inflammatory Drug; RSCCA: Reference centres or Special Centres for Children and Adults; SCD: Sickle-cell disease; VOC: Vaso-occlusive crise; WHO: World Health Organisation.
}

\section{Competing interests}

Authors declare that they have no conflicts of interest. For this project, the University Hospital of Nantes has received funding only from the French health products agency (Agence Nationale de Sécurité du Médicament et des produits de santé - ANSM). The present manuscript has been peerreviewed by a member of this agency.

\section{Authors' contributions}

MG is responsible for the project management. She contributed to the questionnaire development and wrote the first draft of the manuscript. MLC contributed to design the study. She provided her expertise in the area of sickle-cell disease in paediatric patients. MGB provided her expertise in the area of addictology. She contributed to the preparation of the questions of the patient's interview and validated their relevance to evaluate problematic use. FF provided methodological advice, designed the statistical analysis plan and will conduct the statistical analysis. LW conducted literature search and wrote the first draft of the protocol. MR contributed to the questionnaire development and prepared the set-up of the study in the different centres. MLP contributed to the questionnaire development and will conduct the telephone interview. FP contributed to the questionnaire development and will help in analysis and interpretation of data. OB provided his expertise in the area of child and adolescent psychiatry. He contributed to the preparation of the questions of the patient's interview and validated their adequacy with the child's age. MHD provided her expertise in the area of pain management in paediatric patients. PJ read and approved the protocol. CW designed the study and finalized the protocol. All authors read and approved the final manuscript.

\section{Authors' information}

MG is a Pharm D in the Pharmacology Department of Nantes University Hospital, working in the Nantes Centre for Evaluation of and Information on drug Dependence and Addiction monitoring (CEIP-A) and is also an associate member at the SPHERE research team (EA4275) at the Nantes University,

MLC is a MD in the Paediatric Haematology Department of Nantes University Hospital.

MGB is a MD, PhD, psychiatrist in the Addictology and Psychiatry

Department of Nantes University Hospital and a researcher at the SPHERE research team (EA4275)

FF is a methodologist at the Biometry Platform of Nantes University Hospital and a researcher at the SPHERE research team (EA4275).

LW is a MD who worked for the Pharmacology Department at the time of study design. She is now working at the French Blood Establishment.

MR is a clinical research associate in the Clinical Pharmacology Department and in the Addictology and Psychiatry Department of Nantes University Hospital. She is also a researcher at the SPHERE research team (EA4275) at the Nantes University,

MLP is a clinical research associate in the Clinical Pharmacology Department of Nantes University Hospital.

FP is a Pharm D in the Pharmacology Department of Nantes University Hospital, working in the Nantes Centre for Evaluation of and Information on drug Dependence and Addiction monitoring (CEIP-A).

$\mathrm{OB}$ is a MD, psychiatrist, professor and the head of the Child and Adolescent Psychiatry Department, in Nantes University Hospital.

$M H D$ is a MD in the Center for fighting against pain and a pain consultant in Paediatric Oncology and Haematology Department.

$\mathrm{PJ}$ is a MD, PhD, Professor and the head of Clinical Pharmacology

Department. She is also a dean of the Faculty of Medicine and a researcher at the SPHERE research team (EA4275) at the Nantes University.

CW is a Pharm D, PhD in the Clinical Pharmacology Department, working in the Nantes Centre for Evaluation of and Information on drug Dependence and Addiction monitoring (CEIP-A) and a researcher at the SPHERE research team (EA4275) at the Nantes University.

\section{Acknowledgements}

We wish to sincerely thank all the medical practitioners who accepted to participate in the study, for the kindness with which they received the project and for their investment in recruiting patients.

We would also like to thank the French National Agency for Medicines and Health Products Safety (Agence Nationale de Sécurité du Médicament et des produits de santé - ANSM) for the financial support they provided so that 
this research project may be completed, and the Nantes University Hospital for the payment of publication charges of this manuscript.

\section{Author details}

${ }^{1}$ Clinical Pharmacology Department, Nantes University Hospital, Institut de Biologie, 9 quai Moncousu, 44093 Nantes Cedex 1, France. ${ }^{2}$ EA 4275 SPHERE «bioStatistics, Pharmacoepidemiology and Human sciEnces REsearch, Faculties of Medicine and Pharmaceutical Sciences, Nantes University, Nantes, France. ${ }^{3}$ Paediatric Haematology Department, Nantes University Hospital, 7 quai Moncousu, 44093 Nantes Cedex 1, France. ${ }^{4}$ Addictology and Psychiatry Department, Nantes University Hospital, 85 rue de Saint-Jacques, 44093 Nantes Cedex 1, France. ${ }^{5}$ Biometry Platform, Nantes University Hospital, Nantes, France. ${ }^{6}$ Child and Adolescent Psychiatry Department, Nantes University Hospital, 7 quai Moncousu, 44093 Nantes Cedex 1, France. ${ }^{7}$ Paediatric Oncology and Haematology Department, Nantes University Hospital, 7 quai Moncousu, 44093 Nantes Cedex 1, France.

Received: 29 October 2015 Accepted: 8 November 2015

Published online: 14 November 2015

\section{References}

1. Gillman MA. Minireview: analgesic nitrous oxide intercats with the endogenous opioid system: a review of the evidence. Life Sci. 1986;39:1209-21.

2. Gillman MA. Nitrous oxide, an opioid addictive agent. Am J Med. 1986;81:97-102.

3. Berkowitz BA, Finck AD, Ngai SH. Nitrous oxide analgesia: reversal by naloxone and development of tolerance. J Pharmacol Exp Ther. 1977;209:304-8.

4. Hynes MD, Berkowitz BA. Nitrous oxide stimulation of locomotor activity: evidence for an opiate-like behavioral effect. J Pharmacol Exp Ther. 1979;209(2):304-8

5. Wu MS, Hsu YD, Lin JC, Chen SC, Lee JT. Spinal myoclonus in subacute combined degeneration caused by nitrous oxide intoxication. Acta Neurol Taiwan. 2007;16(2):102-5.

6. Waters MF, Kang GA, Mazziotta JC, DeGiorgio CM. Nitrous oxide inhalation as a cause of cervical myelopathy. Acta Neurol Scand. 2005;112(4):270-2.

7. Wagner SA, Clark MA, Wesche DL, Doedens DJ, Lloyd AW. Asphyxial deaths from the recreational use of nitrous oxide. J Forensic Sci. 1992;37(4):1008-15.

8. Waclawik AJ, Luzzio CC, Juhasz-Pocsine K, Hamilton V. Myeloneuropathy from nitrous oxide abuse: unusually high methylmalonic acid and homocysteine levels. WMJ. 2003;102(4):43-5.

9. Suruda AJ, McGlothlin JD. Fatal abuse of nitrous oxide in the workplace. J Occup Med. 1990;32(8):682-4.

10. Sterman AB, Coyle PK. Subacute toxic delirium following nitrous oxide abuse. Arch Neurol. 1983;40(7):446-7.

11. Sethi NK, Mullin P, Torgovnick J, Capasso G. Nitrous oxide "whippit" abuse presenting with cobalamin responsive psychosis. J Med Toxicol. 2006;2(2):71-4.

12. Richardson PG. Peripheral neuropathy following nitrous oxide abuse. Emerg Med Australas. 2010;22(1):88-90.

13. Miller MA, Martinez V, McCarthy R, Patel MM. Nitrous oxide "whippit" abuse presenting as clinical B12 deficiency and ataxia. Am J Emerg Med. 2004;22(2):124.

14. Hwang JC, Himel HN, Edlich RF. Frostbite of the face after recreational misuse of nitrous oxide. Burns. 1996;22(2):152-3.

15. Garbaz L, Mispelaere D, Boutemy M, Jounieaux V. Pneumothorax following recreational inhalation of nitrous oxide. Rev Mal Respir. 2007;24(5):622-4.

16. Chadly A, Marc B, Barres D, Durigon M. Suicide by nitrous oxide poisoning Am J Forensic Med Pathol. 1989;10(4):330-1.

17. Cartner $M$, Sinnott M, Silburn P. Paralysis caused by "nagging". Med J Aust. 2007;187(6):366-7.

18. Alt RS, Morrissey RP, Gang MA, Hoffman RS, Schaumburg HH. Severe myeloneuropathy from acute high-dose Nitrous Oxide (N(2)O) abuse. J Emerg Med. 2011;41(4):378-80.

19. Dohrn CS, Lichtor JL, Coalson DW, Uitvlugt A, de Wit H, Zacny JP. Reinforcing effects of extended inhalation of nitrous oxide in humans. Drug Alcohol Depend. 1993;31(3):265-80.

20. Angoulvant F, Redant S, Holvoet L, Millet B, Ferster A, Andreu-Gallien J. Prise en charge de la douleur des enfants drépanocytaires aux urgences: recommandations et état des lieux dans le Réseau Mère-Enfant de la francophonie. Reanimation. 2011.

21. Haute Autorité de Santé (HAS). Syndromes drépanocytaires majeurs de l'enfant et l'adolescent. Protocole national de diagnostic et de soins pour une maladie rare. Guide médecin Affection Longue Durée, Janvier 2010. http://www.has-sante.fr/portail/upload/docs/application/pdf/2010-04/ald_ 10_pnds_drepano_enfant_web.pdf. Accessed 12 Nov 2015.

22. Midence $L$, Elander J. Adjustement and coping in adults with sickle cell disease: An assessment of research evidence. Br J Health Psychol. 1996;1:95-111.

23. Haute Autorité de Santé (HAS). Syndromes drépanocytaires majeurs de l'adulte. Protocole national de diagnostic et de soins pour une maladie rare. Guide médecin Affection Longue Durée, Janvier 2010. http://www.hassante.fr/portail/upload/docs/application/pdf/2010-04/ald_10_guide_ drepano_adulte_web.pdf. Accessed 12 Nov 2015.

24. Shapiro BS, Benjamin LJ, Payne R, Heidrich G. Sickle cell-related pain: Perceptions of medical practionners. J Pain Symptom Manage. 1997;14:168-74.

25. Waldrop RD, Mandry C. Health professional's perceptions of opioid dependence among patients with pain. Am J Emerg Med. 1995;13:529-31.

26. Fishbain DA, Rosomoff HL, Rosomoff RS. Drug abuse, dependence and addiction in chronic pain patients. Clin J Pain. 1992;8:77-85.

27. Weissman DE, Haddox JD. Opiod pseudoaddiction: An iatrogenic syndrome. Pain. 1989;36:363-6.

28. American Psychiatric Association. Diagnostic and Statistical Manual of Mental Disorders, 4th ed (DSM-IV). Washington DC: American Psychiatric Association; 1994.

29. Elander J, Lusher J, Bevan D, Telfer P. Pain management and symptoms of substance dependence among patients with sickle cell disease. Soc Sci Med. 2003;57:1683-96.

30. Lusher J, Elander J, Bevan D, Telfer P, Burton B. Analgesic addiction and pseudoaddiction in painful chronic illness. Clin J Pain. 2006;22(3):316-24.

31. Elander J, Lusher J, Bevan D, Telfer P, Burton B. Understanding the causes of problematic pain management in sickle cell disease: evidence that pseudoaddiction plays a more important role than guenine analgesic dependence. J Pain Symptom Manag. 2004;27(2):156-69.

32. American Psychiatric Association. Diagnostic and Statistical Manual of Mental Disorders, 5th ed (DSM 5). Washington DC: American Psychiatric Association; 2013.

\section{Submit your next manuscript to BioMed Central and take full advantage of:}

- Convenient online submission

- Thorough peer review

- No space constraints or color figure charges

- Immediate publication on acceptance

- Inclusion in PubMed, CAS, Scopus and Google Scholar

- Research which is freely available for redistribution

Submit your manuscript at www.biomedcentral.com/submit 